\title{
Review
}

\section{Newly discovered angiogenesis inhibitors and their mechanisms of action}

\author{
Ze-hong MIAO*, Jian-ming FENG, Jian DING* \\ Division of Antitumor Pharmacology, State Key Laboratory of Drug Research, Shanghai Institute of Materia Medica, Chinese Academy \\ of Sciences, Shanghai 201203, China
}

\begin{abstract}
In the past decade, the success of angiogenesis inhibitors in clinical contexts has established the antiangiogenic strategy as an important part of cancer therapy. During that time period, we have discovered and reported 17 compounds that exert potent inhibition on angiogenesis. These compounds exhibit tremendous diversity in their sources, structures, targets and mechanisms. These studies have generated new models for further modification and optimization of inhibitory compounds, new information for mechanistic studies and a new drug candidate for clinical development. In particular, through studies on the antiangiogenic mechanism of pseudolaric acid $\mathrm{B}$, we discovered a novel mechanism by which the stability of hypoxia-inducible factor $1 \alpha$ is regulated by the transcription factor c-Jun. We also completed a preclinical study of AL3810, a compound with the potential to circumvent tumor drug resistance to a certain extent. All of these findings will be briefly reviewed in this article.
\end{abstract}

Keywords: cancer therapy; angiogenesis inhibitor; antiangiogenic mechanism; hypoxia-inducible factor $1 \alpha$; pseudolaric acid B; AL3810

Acta Pharmacologica Sinica (2012) 33: 1103-1111; doi: 10.1038/aps.2012.97; published online 27 Aug 2012

\section{Introduction}

Angiogenesis drives tumor progression; in particular, angiogenesis is critical for the growth and metastasis of solid tumors $^{[1-4]}$. The inhibition of tumor angiogenesis has therefore become an important strategy for cancer therapy. Several inhibitors of tumor angiogenesis, such as bevacizumab (Avastin), sorafenib (Nexavar) and sunitinib (Sutent), have been successfully used in the clinic to treat solid tumors ${ }^{[4]}$. This success has led to increased efforts to discover new angiogenesis inhibitors with different mechanisms of action and/or distinct chemical structures. During the past 10 years, we have discovered and reported on 17 compounds that demonstrate potent inhibition of angiogenesis (Table 1). In contrast to the inhibitors that are already employed for clinical purposes, these newly discovered antiangiogenic agents display intriguing diversity in their sources, chemical structures, antiangiogenic mechanisms, and molecular targets. This review will focus on these diverse characteristics of the 17 newly discovered antiangiogenic compounds, as well as the representative findings that have been produced by the mechanistic investigation and preclinical development of these compounds.

\footnotetext{
* To whom correspondence should be addressed.

E-mail zhmiao@mail.shcnc.ac.cn (Ze-hong MIAO); jding@mail.shcnc.ac.cn (Jian DING)

Received 2012-06-15 Accepted 2012-06-19
}

\section{An overview of the 17 antiangiogenic compounds}

In a search for new angiogenesis inhibitors, we performed extensive evaluations of thousands of natural products and synthetic or semi-synthetic compounds over the course of the past 10 years. From this process, 17 compounds were discovered to possess antiangiogenic activity (Table 1). The mechanisms of action of several of these compounds have been investigated, and one of these compounds, namely, AL3810, has been registered as an IND (investigational new drug) for cancer therapy in China.

Analyses of these 17 compounds indicate their diverse sources (Table 1). In particular, 7 of these inhibitors are derived from terrestrial sources, and 5 of these 7 compounds (pseudolaric acid B, triptolide, 10-hydroxycamptothecin, 11,11'-dideoxyverticillin and shiraiachrome A) are used in traditional Chinese medicine (TCM). It is also notable that quercetin is present both in many fruits and vegetables and in olive oil, red wine and tea. Of the remaining 10 compounds that were identified, 5 are marine-derived inhibitors, 3 of which [JG3, MDOS and Grateloupia longifolia polysaccharide (GLP)] are saccharides and 2 of which are saponins from sea cucumber. The final 5 inhibitors are chemically synthesized; these synthesized compounds are also representative of a great diversity of chemical structures, including terpenoids, alkaloids, flavonoids, saccharides, saponins and pyridopyrimidines, as shown in Table 1. 
Table 1. Angiogenesis inhibitors discovered in the Shanghai Institute of Materia Medica since 2001.

\begin{tabular}{l} 
№ Inhibitors \\
\hline $\begin{array}{l}\text { Chemical structure } \\
\text { Pseudolaric } \\
\text { acid } \mathrm{B}\end{array}$
\end{tabular}

2 Triptolide

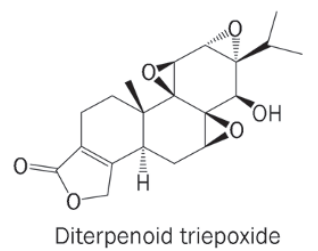

3

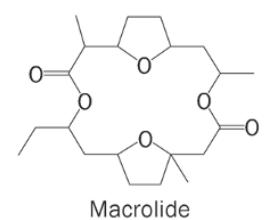

4 10-Hydroxycamptothecin<smiles>CCC1(O)C(=O)OCc2c1cc1n(c2=O)Cc2cc3cc(O)ccc3nc2-1</smiles>

Alkaloid

$5 \quad 11,11^{\prime}$-dideoxyverticillin

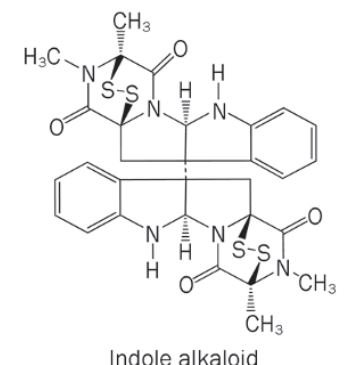

6 Shiraiachrome A
Tripterygium wilfordii XPB; RNAP ॥ Hook F, a TCM

An endophyte Streptomyces sp. Is9131 of Magnolia hookeri

\section{Camptotheca acuminata, a TCM}

Topoisomerase II

\section{The fungus Shiraia VEGF bambusicola, a TCM VEGFR}

The fungus Shiraia bambusicola, a TCM
VEGFR-2; FGFR; PDGFR; EGFR
1. To increase the phosphorylated c-Jun while reducing the non-phosphorylated c-Jun at Ser63/73, which impairs its function in stabilizing HIF- $1 \alpha$;

2. To reduce HIF-1 $\alpha$ protein by promoting its proteasome-mediated degradation;

3. To abrogate hypoxia-induced VEGF secretion via reducing HIF-1 $\alpha$ protein.

1. To increase the levels of HIF-1 $\alpha$ mRNA, but to reduce its transcriptional function;

2. To decrease mRNA levels of HIF-1 $\alpha$ target genes including VEGF, BNIP3, and CAIX;

3. To lower the secretion of VEGF protein, and to reduce sprout outgrowth.

1. To reduce HIF-1 $\alpha$ accumulation, irrelevant to its topoisomerase II inhibition;

2. To abrogate the HIF-1 $\alpha$-driven increase in VEGF MRNA;

3. To reduce constitutive, HIF-1 $\alpha$-independent VEGF secretion and concurrently antagonize inducible, HIF-1 $\alpha$ dependent VEGF secretion.

To inhibit proliferation, migration and tube formation of HMEC cells;

2. To inhibit angiogenesis in CAM assays;

3. To elicit apoptosis in HMEC cells.

1. To antagonize the antiapoptotic effects of VEGF, and to inhibit VEGF-induced HUVEC migration and tube formation;

2. To completely block VEGF-induced microvessel sprouting and vessel growth;

3. To decrease VEGF secretion and to suppress VEGF-induced tyrosine phosphorylation of Flt-1 and KDR/Flk-1.

1. To suppress the autophosphorylation of [7] VEGFR-2, FGFR, PDGFR, and EGFR;

2. To inhibit the proliferation, migration, and tube formation of HMEC;

3. To inhibit the formation of new microvessels in a rat aorta culture model as well as in the CAM assay.

$[20$,

$[16$,

18 , 33]

$$
\text { ], }
$$


№ Inhibitors

Chemical structure

7 Quercetin<smiles>O=c1c(O)c(-c2ccc(O)c(O)c2)oc2cc(O)cc(O)c12</smiles>

Flavonoid

\section{Marine-derived natural products}

8 JG3

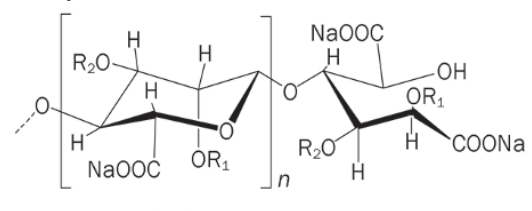

Sulfated oligosaccharide

9 MDOS

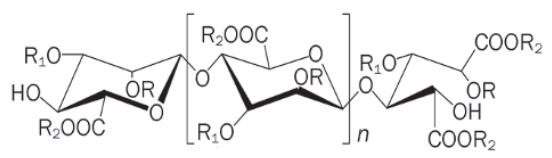

$\mathrm{R}=\mathrm{SO}_{3} \mathrm{Na}, \mathrm{R}_{1}=\mathrm{SO}_{3} \mathrm{Na}$ or $\mathrm{H}$,

$\mathrm{R}_{2}=\mathrm{CH}_{2} \mathrm{CH}(\mathrm{OH}) \mathrm{CH}_{3}$ or $\mathrm{Na}, n=2-8$

Sulfated oligosaccharide

10 Grateloupia Sulphated polysaccharide

longifolia (MW: $1.8 \times 10^{6}$ )

polysac-

charide

11 Philinopside

E

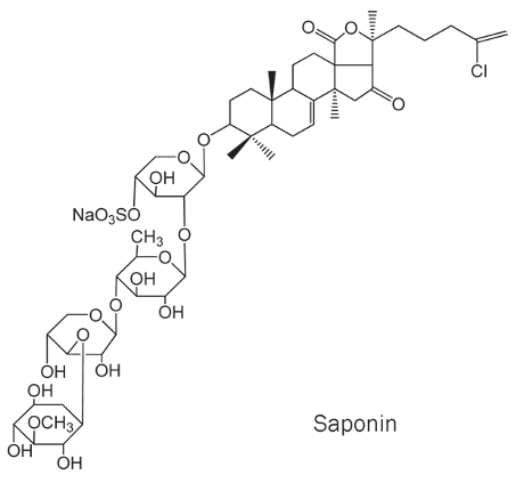

Origin Targets

MMP-2

Many fruits and

vegetables, as

well as olive oil,

red wine, and

tea

Marine oligoman- Heparanase nurarate blocks

Marine oligomannurarate blocks

HER2; EGFR; VEGFR; PDGFR; C-Kit; FGFR1; c-Src

The marine alga Tissue factor G longifolia

Sea cucumber

KDR

(pentacta quadrangularis)
1. To combat heparanase activity via binding to the KKDC and QPLK domains of the heparanase molecule;

2. To abolish heparanase-driven invasion, and to inhibit the release of heparan sulfate-sequestered bFGF from the extracellular matrix, and to repress subsequent angiogenesis;

3. To inactivate bFGF-induced bFGFR and ERK1/2 phosphorylation and to block bFGF-triggered angiogenic events by binding to bFGF.

1. To directly inhibit HER2, EGFR, VEGFR, PDGFR, c-Kit, FGFR1 and c-Src, with little impact on FGFR2;

2. To inhibit phosphorylation of PTKs, exemplified by HER2, EGFR and VEGFR2, and downstream molecules of Erk1/2 and AKT;

3. To act as an ATP competitive inhibitor via directly binding to the residues of entrance rather than those of the ATP-binding pocket.

1. To decrease tissue factor at both mRNA and protein levels;

2. To inhibit proliferation of HMECs and HUVEC and tube formation and to reduce the number of migratory cells in a VEGF-independent manner;

3. To reduce new vessel formation and the vessel density in Matrigel plugs implanted in mice.

1. To inhibit KDR phosphorylation and downstream signaling;

2. To specifically interact with KDR extracellular domain, and to block its interaction with VEGF and the downstream signaling;

3. To markedly suppresses $\alpha \vee \beta 3$ integrin-driven downstream signaling and as a result, to disturb the physical interaction between KDR and $\alpha \vee \beta 3$ integrin in HMECs, followed by disruption of the actin cytoskeleton organization and decreased cell adhesion to vitronectin.
[27, 


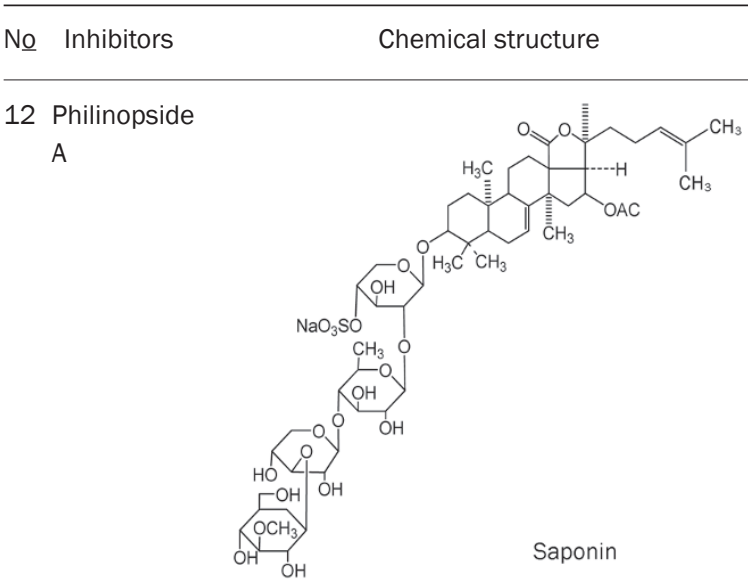

Synthetic inhibitors

13 AL3810<smiles>COc1cc2c(Oc3ccc4c(C(C)=O)cccc4c3)ccnc2cc1OCC1(N)CC1</smiles>

$14 \mathrm{BB}$<smiles>COc1cc2ncnc(Nc3cccc(Br)c3)c2cc1OCCCN1CCC(OC)C1</smiles>

Uinonazoline

15 TKI-28<smiles>Cn1c(=O)c(-c2c(Cl)cccc2Cl)cc2cnc(Nc3ccccc3)nc21</smiles>

16 TKI-31<smiles>Cn1c(=O)c(-c2c(Cl)cccc2Cl)cc2cnc(Nc3cccc(F)c3)nc21</smiles>

Sea cucumber (pentacta quadrangularis)

Origin

Targets

Antiangiogenic mechanisms

Refs

VEGFR; FGFR1; 1 . To inhibit the proliferation, migration PDGFRß; EGFR and tube formation of HMECs;

2. To suppress the formation of new microvessels in cultured rat aorta and angiogenesis in CAM assays;

3. To inhibit VEGFR, FGFR1, PDGFR $\beta$, and EGFR.

[10] $\begin{array}{ll}\text { Synthetic } & \text { VEGFR1; } \\ \text { VEGFR2; } & \text { FGFR1; } \\ \text { PDGFR } \beta\end{array}$

Synthetic

EGFR

Synthetic

Synthetic
VEGFR2;

PDGFRß; c-kit;

C-SrC
1. To inhibit VEGFR1, VEGFR2, FGFR1 and PDGFRB;

2. To inhibit the autophosphorylation of VEGFR2, PDGFR $\beta$, and FGFR1 in endothelial cells;

3. To exhibit potent antiangiogenesis activity, manifested by significant inhibition of microvessel outgrowth of rat arterial ring and CAM in ex vivo angiogenesis models.

1. To selectively inhibit EGFR;

2. To abrogate autophosphorylation of the EGF-stimulated EGFR and phosphorylation of its key downstream signaling molecules ERK and AKT in A549 cells;

3. To exhibit antiangiogenesis activity, as evidenced by antagonizing EGFinduced HMECS migration in vitro, blocking HMECS tube formation, and inhibiting microvessel sprouting from rat aortic rings.

ErbB-2; EGFR; KDR; PDGFRß; c-kit; c-Src

1. To inhibit ErbB-2, EGFR, KDR, PDGFR $\beta$, c-kit and c-Src in cell-free systems;

2. To block their autophosphorylation and subsequently to downregulate phosphorylation of many downstream signaling proteins at the cellular level;

3. To inhibit cell proliferation driven by EGF, VEGF and PDGF, and cell migration and tube formation in HMECs.

1. To inhibit VEGFR2, PDGFRß, c-kit and $c-S r c$, showing no activity against VEGFR1 and EGFR;

2. To repress VEGF-induced phosphorylation of VEGFR2 in endothelial cells and PDGFBB-induced phosphorylation in fibroblast cells, leading to the inhibition of PI3K/Akt/mTOR, MAPK42/44 (ERK) and paxillin;
$[11$, 31] 


№ Inhibitors Chemical structure Origin

3. To suppress VEGF-induced endothelial cells proliferation, migration and their differentiation into capillarylike tube formation.

\section{$17 \quad \mathrm{Cg}$}

1. To inhibit proliferation, migration and tube formation of endothelial cells, and angiogenesis in aortic ring and CAM assays;

2. To induce disassembly of microtubules in endothelial cells and to downregulate Raf-MEK-ERK signaling activated by pro-angiogenic factors;

3. To disrupt capillary-like networks and newly formed vessels in vitro and to rapidly decrease perfusion of neovasculature in vivo, and to induce endothelial cell contraction and membrane blebbing in neovasculature dependent on the Rho/Rho kinase pathway.

Abbreviations: BNIP3, BCL2/adenovirus E1B 19 kDa protein-interacting protein 3; CAIX, carbonic anhydrase IX; CAM, chick chorioallantoic membrane; EGFR, epidermal growth factor receptor; ERK, extracellular signal regulated kinase; bFGF, basic fibroblast growth factor; FGFR1, FGF receptor 1; HER2, human epidermal growth factor receptor-2; HIF-1 $\alpha$, hypoxia-inducible factor 1alpha; HMEC, human dermal microvasculature endothelial cells; HUVEC, human umbilical vein endothelial cells; KDR, receptor for vascular endothelial growth factor; MEK, MAPK/ERK kinase; MMP-2, matrix metalloproteinase-2; PDGFBB, platelet derived growth factor BB; PDGFR, platelet-derived growth factor receptor; RTK, receptor tyrosine kinase; VEGF, vascular endothelial growth factor; VEGFR, vascular endothelial growth factor receptor; TCM, traditional Chinese medicine.

The apparent differences in the sources and chemical structures of the 17 inhibitors are reflected in their distinct (potential) targets. These targets include angiogenic kinases (angiokinases), extracellular matrix (ECM) components and the hypoxia-inducible factor 1 $\alpha$ (HIF-1 $\alpha$ )-vascular endothelial growth factor (VEGF) axis, among others (Table 1, Figure 1). Moreover, the antiangiogenic activities of these compounds revealed in the in vitro, ex vivo and/or in vivo experimental models are mediated by distinct molecular signaling pathways (Figure 1).

\section{Angiokinase inhibitors}

Although many protein tyrosine kinases (PTKs) contribute to the angiogenic process, the VEGF-VEGF receptor (VEGFR) axis is the main target for clinical applications of antiangiogenic therapy ${ }^{[5]}$. Of the 17 compounds listed in Table 1, 9 compounds, namely, 11,11'-dideoxyverticillin, shiraiachrome A, MDOS, philinopside A, philinopside E, AL3810, BB, TKI-28, and TKI-31, were found to directly inhibit this axis ${ }^{[6-14]}$ (Table 1 and Figure 1). The former 5 compounds are derived from natural products, whereas the latter 4 inhibitors are synthetic. These 9 compounds display different profiles of PTK inhibition, as they have distinct selectivity against various receptor and/or non-receptor tyrosine kinases, including human epi-

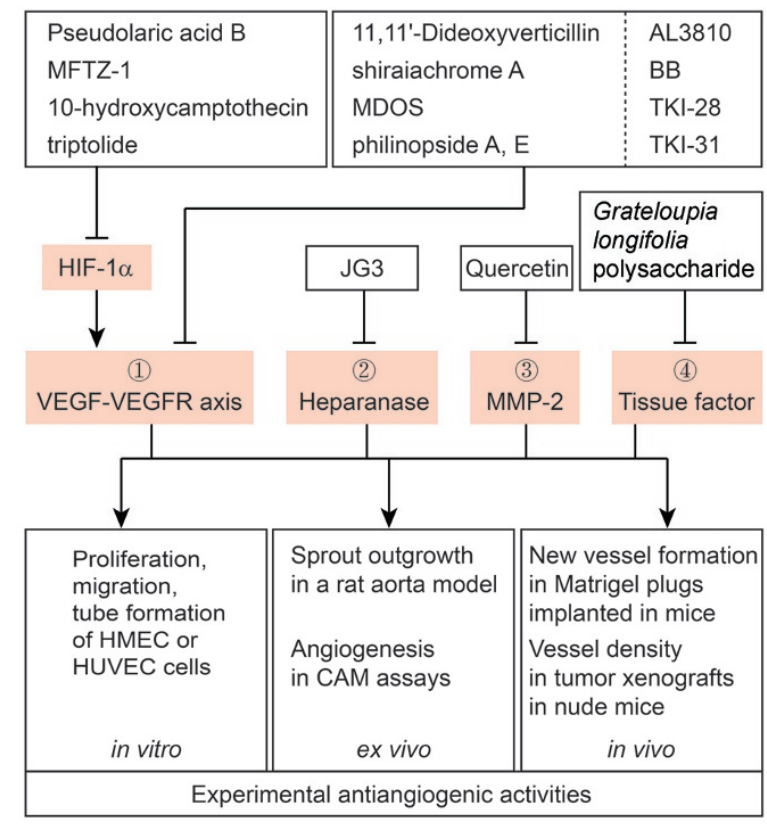

Figure 1. A schematic overview of molecular signaling that possibly mediates experimental antiangiogenic activities of the compounds discussed here. 
dermal growth factor receptor 2 (HER2), epidermal growth factor receptor (EGFR), VEGFR, platelet-derived growth factor receptor (PDGFR), c-Kit, fibroblast growth factor receptor 1 (FGFR1) and/or c-Src. Nevertheless, all 9 of these compounds can directly suppress the critical angiokinase VEGFR, producing significant experimentally observed antiangiogenic effects as a result (Table 1 and Figure 1). In particular, AL3810 has been in clinical trials because it not only displays excellent anticancer and antiangiogenic activities but also demonstrates good pharmacokinetics and toxicity in preclinical studies ${ }^{[15,16]}$.

\section{HIF-1 $\alpha$-VEGF axis inhibitors}

We also found that of the 17 identified antiangiogenic compounds, 4 compounds, namely, pseudolaric acid B, MFTZ-1, 10-hydroxycamptothecin and triptolide, can indirectly inhibit the VEGF-VEGFR axis by decreasing cellular HIF-1a accumulation and thereby reducing VEGF expression and secretion $^{[15-21]}$ (Figure 1). We define these compounds to be HIF-1aVEGF axis inhibitors. HIF-1a is a critical transcription factor that impacts tumor angiogenesis by regulating the expression of VEGF. HIF-1a has thus been proposed as a promising anticancer target.

The HIF-1a-VEGF axis inhibitors reduce the cellular amount of HIF-1a in different ways. Pseudolaric acid B targets microtubulin and causes its depolymerization ${ }^{[22,23]}$. Pseudolaric acid $B$ inhibits angiogenesis by reducing the stability of HIF-1a and thereby downregulating the VEGF-VEGFR axis ${ }^{[15,16,18]}$. However, there has been no direct evidence indicating any association between its antiangiogenic activity and its inhibition of microtubulin ${ }^{[23,24]}$. By contrast, MFTZ-1 does not affect either the degradation of HIF-1a protein or the level of HIF-1a mRNA. Instead, MFTZ-1 can abrogate the HIF-1adriven increase in VEGF mRNA and VEGF protein secretion, producing antiangiogenic effects. Specifically, MFTZ-1 can reduce constitutive, HIF-1a-independent VEGF secretion and concurrently antagonize inducible, HIF-1a-dependent VEGF secretion, in an effect that is independent of its inhibition of its primary target, topoisomerase $\mathrm{II}^{[17,25]}$. The inhibition of angiogenesis by 10 -hydroxycamptothecin ${ }^{[19]}$ may be associated with this drug's suppression of HIF-1a expression, which occurs via the repression of topoisomerase I-dependent transcription. This mechanism of action is possibly similar to the mechanism that is used by topotecan, another camptothecin derivative ${ }^{[26]}$. In contrast to the previous 3 inhibitors, triptolide may enhance the levels of cellular HIF-1a mRNA and protein ${ }^{[20]}$. However, triptolide also causes the downregulation of VEGF expression and secretion ${ }^{[20]}$, possibly because it binds to XPB (which is also known as ERCC3) and causes the degradation of RNA polymerase $\mathrm{II}^{[21]}$, disrupting the transcriptional function of HIF-1a.

\section{ECM component inhibitors}

ECM components including heparanase and matrix metalloproteinase (MMP) are critically involved in the metastatic and angiogenic capabilities of tumor cells. Inhibitors targeting ECM components are increasingly emerging as promising agents for cancer therapy. We have discovered a series of compounds that inhibit tumor angiogenesis by targeting heparanase and MMP. Oligomannurarate sulfate (JG3), a novel oligosaccharide, was identified as a heparanase inhibitor. JG3 significantly inhibits tumor angiogenesis and metastasis, both in vitro and in vivo, by combating heparanase activity; in particular, this effect is mediated through the binding of JG3 to the KKDC and QPLK domains of heparanase. In addition, JG3 abolished heparanase-driven invasion, inhibited the release of basic fibroblast growth factor (bFGF) from the ECM and repressed subsequent angiogenesis ${ }^{[27]}$. By contrast, the antiangiogenic effects of quercetin were found to be associated with its downregulation of MMP-2 ${ }^{[28]}$. In fact, however, other studies reveal complicated molecular mechanisms involved in the antiangiogenic activity of quercetin, including its inhibition of the synthesis and accumulation of the HIF-1a protein; this inhibition reduces the production and secretion of VEGF, as discussed above ${ }^{[29]}$.

\section{Other angiogenesis inhibitors}

C9 and GLP exert antiangiogenic effects through relatively unique mechanisms of action (Table 1). C9 is a new microtubule-depolymerizing agent. In striking contrast to pseudolaric acid B, C9 elicits its antiangiogenic and vascular disrupting effects by inducing microtubule disassembly, the downregulation of Raf-MEK-ERK signaling and the reorganization of actin through the Rho/Rho kinase pathway ${ }^{[24]}$. GLP is a new type of polysaccharide isolated from the alga G longifolia. GLP causes obvious in vitro and in vivo antiangiogenic effects that are not associated with classical VEGF-VEGFR signaling. GLP decreases tissue factor at both the mRNA and protein levels, and this effect may be involved in the inhibition of angiogenesis by GLP ${ }^{[30]}$.

Our findings provide the following beneficial clues to facilitate the future discovery of angiogenesis inhibitors: (1) Natural products are an important source of angiogenesis inhibitors. In particular, through drug discovery guided by therapeutic experiences from long-established TCM practices, inhibitors of angiogenesis could be obtained that have new chemical structures and unique mechanisms of action; examples of these sorts of inhibitors include pseudolaric acid B, triptolide, 11,11'-dideoxyverticillin and shiraiachrome A (Table 1). The original medicinal materials producing these compounds have long been used to either treat various angiogenesisrelated diseases, such as rheumatoid arthritis, microbial skin diseases and psoriasis ${ }^{[7,21,23]}$, or cause the early termination of pregnancies ${ }^{[23]}$. (2) Marine-derived compounds could be another important source of angiogenesis inhibitors, and certain compounds with specific types of chemical structures, such as saccharides, should be subjected to particular scrutiny; as demonstrated by JG3 ${ }^{[27]}$ and GLP ${ }^{[30]}$, these compounds may exhibit unique modes of antiangiogenic activity. (3) Chemical synthesis, particularly if it is based on rational designs and modifications, is a common, frequently necessary method of generating compounds with possible medical applications; this principle is demonstrated by AL3810 in this instance ${ }^{[11,31]}$ 
and is generally exemplified by a variety of clinically utilized drugs. (4) The compounds that we have discovered (Table 1) can be used as chemical models for further modification and optimization to improve their therapeutic potential as angiogenesis-inhibiting drugs for clinical use. (5) The known primary target(s) of certain compounds, such as pseudolaric acid $\mathrm{B}^{[15,16,18]}$ and MFTZ-1 ${ }^{[17]}$, are not necessarily related to their antiangiogenic effects, indicating that unknown and potentially novel mechanism(s) are involved in these effects; these mechanisms merit further investigation.

\section{The discovery that the stability of HIF-1 $\alpha$ protein is regulated by $c$-Jun based on mechanistic studies of the antiangiogenic activity of pseudolaric acid $B$}

Pseudolaric acid B is a diterpenoid isolated from the root bark of Pseudolarix amabilis ${ }^{[32]}$. Pseudolaric acid B has been demonstrated to both elicit potent anticancer effects by depolymerizing microtubulin ${ }^{[22,23]}$ and circumvent tumor multidrug resistance $^{[22]}$. Detailed structure-activity studies have revealed that the components of pseudolaric acid B that are essential to its anticancer activity include a hydrophobic group $\left(-\mathrm{CO}_{2} \mathrm{Me}\right.$ or -Me) at C-7, a $\Delta^{7}$ double bond, an acyloxy (OAc) at C-4,3 and a side chain with a conjugated double bond and a hydrophilic terminal group ${ }^{[33]}$. Based on its traditional use in Chinese folk medicine for facilitating the early termination of pregnancy, we first discovered and reported that its antiangiogenic activity occurred because it accelerates the proteasome-executed degradation of the HIF-1a protein ${ }^{[18]}$.

We previously reported that the activation of the transcription factor c-Jun plays a critical role in the circumvention of tumor multidrug resistance by salvicine ${ }^{[34,35]}$. Based on those earlier findings, we investigated the effect of pseudolaric acid $B$ and found that it could also drive c-Jun phosphorylation ${ }^{[16]}$. During our attempts to correlate HIF-1a protein degradation with the c-Jun phosphorylation induced by pseudolaric acid $\mathrm{B}$, a novel mechanism was revealed in which c-Jun in its nonphosphorylated form regulates the stability of the HIF-1a protein $^{[15,16]}$ (Figure 2).

HIF-1a is a transcription factor that drives neoangiogenesis by regulating the expression of various target genes, including the proangiogenic genes VEGF and VEGFR, in response to hypoxia during the growth of solid tumors ${ }^{[36]}$. The ubiquitination-mediated, proteasome-executed degradation constitutes a critical methanism of regulating the stability of the cellular HIF-1a protein $^{[37]}$. HIF-1a can be hydroxylated by an oxygensensitive prolyl hydroxylase at the Pro402 and Pro564 residues within its oxygen-dependent degradation domain (ODD) ${ }^{[38]}$. This hydroxylation will promote the ubiquitination of HIF-1a at Lys532, a process that is effectively mediated by the ubiquitin ligase known as the Von Hippel-Lindau tumor suppressor $(\mathrm{pVHL})^{[39]}$. This ubiquitination then, in turn, leads to the reduced stability of HIF-1a by facilitating its degradation through the 26S proteasome ${ }^{[15]}$ (Figure 2).

Our studies demonstrated that c-Jun binds to the ODD of HIF-1a protein, protecting HIF-1a from being ubiquitinated and thereby enhancing its stability by reducing its suscep-

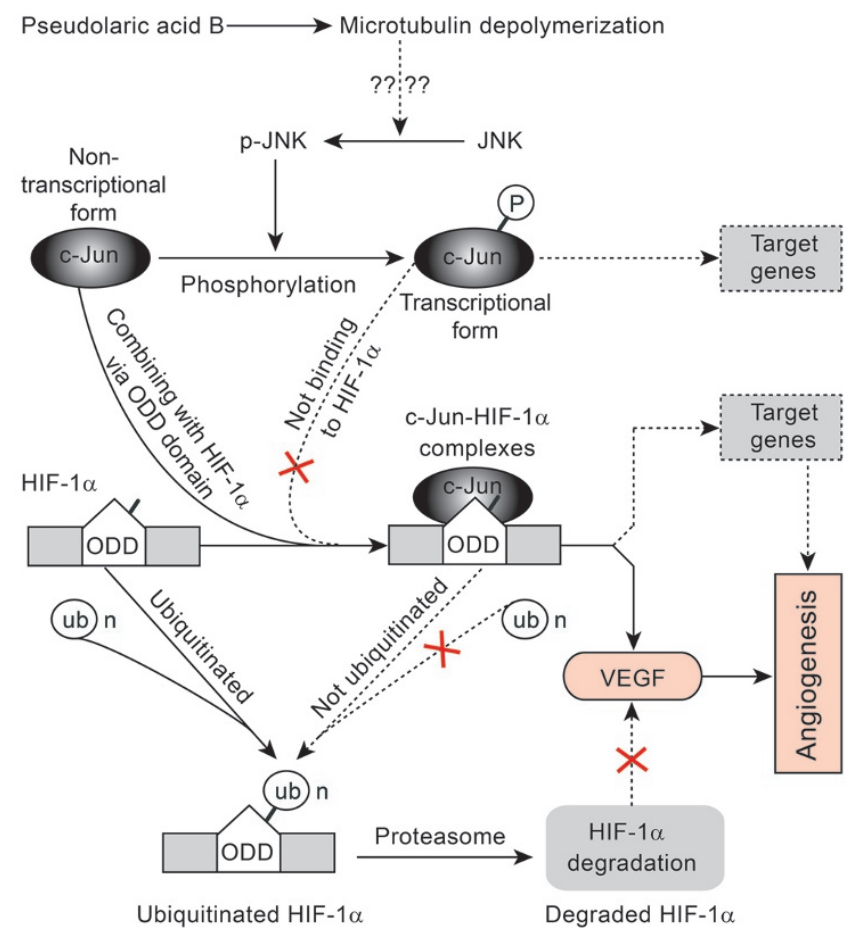

Figure 2. The antiangiogenic mechanism of pseudolaric acid B. Solid or broken lines indicate the relationships between the linked factors with direct experimental evidence (solid lines) or with logical possibility (broken lines) in the case of pseudolaric acid B.

tibility to the proteasome-executed degradation ${ }^{[15]}$ (Figure 2). Notably, this binding requires the domains of c-Jun for DNA binding and heterodimerization but is independent of Ser63/73 phosphorylation. Further investigations have clarified that only non-phosphorylated c-Jun, ie, c-Jun without transcriptional activity, can bind to and protect HIF-1a ${ }^{[15,16]}$. Relatively constant total levels of c-Jun are generally maintained within the cell. Pseudolaric acid B causes the increased phosphorylation of c-Jun, thus reducing the proportion of c-Jun that is in the Ser63/73-non-phosphorylated form. Consequently, the quantity of c-Jun that is able to bind to HIF-1a decreases, impairing the ability of c-Jun to stabilize HIF-1a ${ }^{[16]}$ (Figure 2). Our findings constituted the first discovery of a function for the non-phosphorylated form of c-Jun; in accordance with these results, a function-converter model of c-Jun was proposed, in which Ser63/73 phosphorylation serves as a function converter that shifts c-Jun from its non-transcriptional functions to its transcriptional functions ${ }^{[16]}$.

\section{The development of AL3810 as a clinically promising angiokinase inhibitor}

Various PTKs are aberrantly activated during tumor progression. Several of these PTKs, including VEGFR, PDGFR, and FGFR1, have been demonstrated to contribute to tumor angiogenesis $^{[11,31]}$. In recent years, we have discovered hundreds of compounds with inhibitory activities against different PTKs and have reported that 9 compounds exhibit potent antiangio- 
genic effects (Table 1). Of these 9 compounds, AL3810 (also designated as E-3810) ${ }^{[31]}$ demonstrates the greatest potential for clinical use.

AL3810 is a synthetic multitargeted PTK inhibitor that inhibits VEGFR1, VEGFR2, PDGFRa, PDGFR $\beta$, and FGFR1 with $\mathrm{IC}_{50}$ values in the nanomolar range ${ }^{[11,31]}$. In endothelial cells, AL3810 can suppress the autophosphorylation of VEGFR2, PDGFR $\beta$ and FGFR1. AL3810 thus displays apparent antiangiogenic activity in all of the tested in vitro, ex vivo, and in vivo models (Figure 1). Even at millimolar concentrations, however, AL3810 demonstrates no cytotoxic effects on cancer cell lines. Nevertheless, AL3810 can elicit broad-spectrum in vivo antitumor activity in human kidney, pancreas and liver cancer xenograft models; its activity appears to make it more potent than several commercially available multitargeted PTK inhibitors, such as sorafenib and Sutent. Moreover, its antitumor activity is closely correlated with its antiangiogenic activity $^{[11,31]}$.

Notably, it appears that tumors do not easily become resistant to AL3810, as xenografted tumors re-grown after the withdrawal of AL3810 demonstrate a response to a second cycle of AL3810 treatment that is similar to the response observed for the first treatment cycle. By contrast, tumors re-grown after the withdrawal of sunitinib treatment display reduced sensitivity to a second cycle of treatment with sunitinib. However, these sunitinib-resistant tumors remain sensitive to AL3810 ${ }^{[31]}$. Although this result requires further confirmation, particularly in the clinical context, and the mechanism underlying this result also must be clarified, its potential importance is obvious, particularly given that tumors are generally prone to becoming resistant to the PTK inhibitors that are currently in clinical use ${ }^{[40]}$.

The oral bioavailability of AL3810 is excellent, reaching 31\% in rats ${ }^{[11]}$. Moreover, the concentration of AL3810 in different tissues is higher than the concentration of AL3810 in the plasma of tumor-bearing nude mice. In particular, a 51.7-fold increase in the concentration of AL3810 was detected in the examined tumoral tissues when compared with that in plasma, demonstrating the high affinity of AL3810 to tumors ${ }^{[11]}$. In addition, AL3810 has a relatively long terminal half-life of approximately $4 \mathrm{~h}$; this extended half-life may help explain its persistent antitumor effects ${ }^{[31]}$. All of these results indicate that AL3810 possesses a favorable pharmacokinetic profile.

The prominent advantages of AL3810, which include its potent antiangiogenic effect, its broad spectrum of antitumor activity, its ability to potentially circumvent the drug resistance of tumors and its favorable pharmacokinetic profile, make it an excellent candidate for development as an anticancer drug. AL3810 has already entered into clinical trials in Europe to assess its potential use for this application, and its clinical trials in China will be launched shortly ${ }^{[11,31]}$.

\section{Concluding remarks}

As an aspect of international efforts to explore new antiangiogenic compounds, we investigated the inhibition of angiogenesis by 17 compounds. These compounds have diverse origins, structures, primary targets and mechanisms. Our findings provide new models for the further modification and optimization of antiangiogenic compounds, and new clues related to the examination of antiangiogenic mechanisms. Tumor drug resistance poses a challenge for the antiangiogenic agents that are in current use ${ }^{[4]]}$. Therefore, one of the most urgent tasks in the future will be to both demonstrate the critical molecular mechanism(s) underlying the effects of AL3810 and accelerate the clinical development of AL3810 to meet the potential clinical need for agents that can circumvent this tumor drug resistance. The detailed dissection of the structure-effect relationships of certain compounds to analyze the relationships between their antiangiogenic mechanisms and their cytotoxicity and between their primary targets and their antiangiogenic mechanisms could be another important task to accomplish, as well; the elucidation of these relationships could generate new strategies for cancer therapy.

\section{Acknowledgements}

The authors sincerely thank all of the individuals who have contributed to the work that has been reviewed in this article, including all of the faculty members, postdoctors and students who were either directly or indirectly involved in antiangiogenic studies in the Division of Antitumor Pharmacology, as well as all of our collaborators in the Natural Products Chemistry and Medicinal Chemistry fields who provided us with the compounds that were used in the studies that have been reviewed in this paper.

This work was supported by grants from the National Natural Science Foundation of China (№ 81025020 and № 81021062), the National Basic Research Program of China (№ 2012CB932502), the National Science \& Technology Major Project of China (№ 2012ZX09301-001-002) and the "Interdisciplinary Cooperation Team" Program for Science and Technology Innovation of the Chinese Academy of Sciences.

\section{References}

1 Zetter BR. The scientific contributions of M. Judah Folkman to cancer research. Nat Rev Cancer 2008; 8: 647-54.

2 Eichhorn ME, Kleespies A, Angele MK, Jauch KW, Bruns CJ. Angiogenesis in cancer: molecular mechanisms, clinical impact. Langenbecks Arch Surg 2007; 392: 371-9.

3 Fischer C, Mazzone M, Jonckx B, Carmeliet P. FLT1 and its ligands VEGFB and PIGF: drug targets for anti-angiogenic therapy? Nat Rev Cancer 2008; 8: 942-56.

4 Argyriou AA, Giannopoulou E, Kalofonos HP. Angiogenesis and antiangiogenic molecularly targeted therapies in malignant gliomas. Oncology 2009; 77: 1-11.

5 Tugues S, Koch S, Gualandi L, Li X, Claesson-Welsh L. Vascular endothelial growth factors and receptors: anti-angiogenic therapy in the treatment of cancer. Mol Aspects Med 2011; 32: 88-111.

6 Chen Y, Zhang YX, Li MH, Zhao WM, Shi YH, Miao ZH, et al. Antiangiogenic activity of $11,11^{\prime}$-dideoxyverticillin, a natural product isolated from the fungus Shiraia bambusicola. Biochem Biophys Res Commun 2005; 329: 1334-42.

7 Tong $\mathrm{Y}$, Zhang X, Zhao W, Zhang Y, Lang J, Shi Y, et al. Anti-angiogenic effects of Shiraiachrome A, a compound isolated from a Chinese folk medicine used to treat rheumatoid arthritis. Eur J Pharmacol 2004; 
494: 101-9.

8 Ma J, Xin X, Meng L, Tong L, Lin L, Geng M, et al. The marine-derived oligosaccharide sulfate (MdOS), a novel multiple tyrosine kinase inhibitor, combats tumor angiogenesis both in vitro and in vivo. PLoS One 2008; 3: e3774.

9 Tian F, Zhu CH, Zhang XW, Xie X, Xin XL, Yi YH, et al. Philinopside E, a new sulfated saponin from sea cucumber, blocks the interaction between kinase insert domain-containing receptor (KDR) and alphavbeta3 integrin via binding to the extracellular domain of KDR. Mol Pharmacol 2007; 72: 545-52.

10 Tong Y, Zhang X, Tian F, Yi Y, Xu Q, Li L, et al. Philinopside A, a novel marine-derived compound possessing dual anti-angiogenic and antitumor effects. Int J Cancer 2005; 114: 843-53.

11 Zhou Y, Chen Y, Tong L, Xie H, Wen W, Zhang J, et al. AL3810, a multityrosine kinase inhibitor, exhibits potent anti-angiogenic and antitumor activity via targeting VEGFR, FGFR, and PDGFR. J Cell Mol Med 2012. doi: 10.1111/j.1582-4934.2012.01541.x.

12 Sun QM, Miao ZH, Lin LP, Gui M, Zhu CH, Xie H, et al. BB, a new EGFR inhibitor, exhibits prominent anti-angiogenesis and antitumor activities. Cancer Biol Ther 2009; 8: 1640-7.

13 Guo XN, Zhong L, Tan JZ, Li J, Luo XM, Jiang HL, et al. In vitro pharmacological characterization of TKI-28, a broad-spectrum tyrosine kinase inhibitor with anti-tumor and anti-angiogenic effects. Cancer Biol Ther 2005; 4: 1125-32.

14 Zhong L, Guo XN, Zhang XH, Sun QM, Tong $\sqcup$, Wu ZX, et al. TKI-31 inhibits angiogenesis by combined suppression signaling pathway of VEGFR2 and PDGFRbeta. Cancer Biol Ther 2006; 5: 323-30.

15 Yu B, Miao ZH, Jiang Y, Li MH, Yang N, Li T, et al. c-Jun protects hypoxia-inducible factor-1alpha from degradation via its oxygen-dependent degradation domain in a nontranscriptional manner. Cancer Res 2009; 69: 7704-12.

16 Yu B, Li MH, Wang W, Wang YQ, Jiang Y, Yang SP, et al. Pseudolaric acid B-driven phosphorylation of C-Jun impairs its role in stabilizing HIF-1alpha: a novel function-converter model. J Mol Med (Berl) 2012; 90: 971-81.

17 Dai M, Miao ZH, Ren X, Tong $\sqcup$, Yang N, Li T, et al. MFTZ-1 reduces constitutive and inducible HIF-1alpha accumulation and VEGF secretion independent of its topoisomerase II inhibition. J Cell Mol Med 2010; 14: 2281-91.

18 Li MH, Miao ZH, Tan WF, Yue JM, Zhang C, Lin LP, et al. Pseudolaric acid $B$ inhibits angiogenesis and reduces hypoxia-inducible factor 1alpha by promoting proteasome-mediated degradation. Clin Cancer Res 2004; 10: 8266-74.

19 Xiao D, Tan W, Li M, Ding J. Antiangiogenic potential of 10-hydroxycamptothecin. Life Sci 2001; 69: 1619-28.

20 Zhou ZL, Luo ZG, Yu B, Jiang Y, Chen Y, Feng JM, et al. Increased accumulation of hypoxia-inducible factor-1alpha with reduced transcriptional activity mediates the antitumor effect of triptolide. Mol Cancer 2010; 9: 1-11.

21 Zhou ZL, Yang YX, Ding J, Li YC, Miao ZH. Triptolide: structural modifications, structure-activity relationships, bioactivities, clinical development and mechanisms. Nat Prod Rep 2012; 29: 457-75.

22 Wong VK, Chiu P, Chung SS, Chow LM, Zhao YZ, Yang BB, et al. Pseudolaric acid $B$, a novel microtubule-destabilizing agent that circumvents multidrug resistance phenotype and exhibits antitumor activity in vivo. Clin Cancer Res 2005; 11: 6002-11.

23 Tong YG, Zhang XW, Geng MY, Yue JM, Xin XL, Tian F, et al. Pseudolarix acid $B$, a new tubulin-binding agent, inhibits angiogenesis by interacting with a novel binding site on tubulin. Mol Pharmacol 2006; 69: 1226-33.
24 Ren X, Dai M, Lin LP, Li PK, Ding J. Anti-angiogenic and vascular disrupting effects of $\mathrm{C}$, a new microtubule-depolymerizing agent. $\mathrm{Br}$ J Pharmacol 2009; 156: 1228-38.

25 Xie CY, Zhu H, Lin LP, Miao ZH, Geng MY, Cai YJ, et al. MFTZ-1, an actinomycetes subspecies derived antitumor macrolide, functions as a novel topoisomerase II poison. Mol Cancer Ther 2007; 6: 3059-70.

26 Kummar S, Raffeld M, Juwara L, Horneffer Y, Strassberger A, Allen D, et al. Multihistology, target-driven pilot trial of oral topotecan as an inhibitor of hypoxia-inducible factor-1alpha in advanced solid tumors. Clin Cancer Res 2011; 17: 5123-31.

27 Zhao H, Liu H, Chen Y, Xin X, Li J, Hou Y, et al. Oligomannurarate sulfate, a novel heparanase inhibitor simultaneously targeting basic fibroblast growth factor, combats tumor angiogenesis and metastasis. Cancer Res 2006; 66: 8779-87.

28 Tan WF, Lin LP, Li MH, Zhang YX, Tong YG, Xiao D, et al. Quercetin, a dietary-derived flavonoid, possesses antiangiogenic potential. Eur J Pharmacol 2003; 459: 255-62.

29 Lee DH, Lee YJ. Quercetin suppresses hypoxia-induced accumulation of hypoxia-inducible factor-1alpha (HIF-1alpha) through inhibiting protein synthesis. J Cell Biochem 2008; 105: 546-53.

30 Zhang C, Yang F, Zhang XW, Wang SC, Li MH, Lin LP, et al. Grateloupia longifolia polysaccharide inhibits angiogenesis by downregulating tissue factor expression in HMEC-1 endothelial cells. $\mathrm{Br} J$ Pharmacol 2006; 148: 741-51.

31 Bello E, Colella G, Scarlato V, Oliva P, Berndt A, Valbusa G, et al. E-3810 is a potent dual inhibitor of VEGFR and FGFR that exerts antitumor activity in multiple preclinical models. Cancer Res 2011; 71: 1396-405.

32 Chiu P, Leung LT, Ko BC. Pseudolaric acids: isolation, bioactivity and synthetic studies. Nat Prod Rep 2010; 27: 1066-83.

33 Yang SP, Cai YJ, Zhang BL, Tong LJ, Xie H, Wu Y, et al. Structural modification of an angiogenesis inhibitor discovered from traditional Chinese medicine and a structure-activity relationship study. J Med Chem 2008; 51: 77-85.

34 Miao ZH, Ding J. Transcription factor c-Jun activation represses $m d r-1$ gene expression. Cancer Res 2003; 63: 4527-32.

35 Miao ZH, Tang T, Zhang YX, Zhang JS, Ding J. Cytotoxicity, apoptosis induction and downregulation of MDR-1 expression by the antitopoisomerase II agent, salvicine, in multidrug-resistant tumor cells. Int J Cancer 2003; 106: 108-15.

36 Weidemann A, Johnson RS. Biology of HIF-1alpha. Cell Death Differ 2008; 15: 621-7.

37 Semenza GL. Hydroxylation of HIF-1: oxygen sensing at the molecular level. Physiology (Bethesda) 2004; 19: 176-82.

38 Epstein AC, Gleadle JM, McNeill LA, Hewitson KS, O'Rourke J, Mole DR, et al. C elegans EGL-9 and mammalian homologs define a family of dioxygenases that regulate HIF by prolyl hydroxylation. Cell 2001; 107: 43-54.

39 Berra E, Ginouves A, Pouyssegur J. The hypoxia-inducible-factor hydroxylases bring fresh air into hypoxia signalling. EMBO Rep 2006; 7: $41-5$.

40 Rosenzweig SA. Acquired resistance to drugs targeting receptor tyrosine kinases. Biochem Pharmacol 2012; 83: 1041-8.

41 Bergers G, Hanahan D. Modes of resistance to anti-angiogenic therapy. Nat Rev Cancer 2008; 8: 592-603.

42 Li QN, Liu HY, Xin XL, Pan QM, Wang L, Zhang J, et al. Marine-derived oligosaccharide sulfate (JG3) suppresses heparanase-driven cell adhesion events in heparanase over-expressing CHO-K1 cells. Acta Pharmacol Sin 2009; 30: 1033-8. 